Revue pluridisciplinaire d'études médiévales

\title{
La gloire de Dieu, la gloire du prince. Manifestations divines et légitimation dans le duché de Bretagne au XIV siècle
}

David Dominé-Cohn

\section{(2) OpenEdition}

\section{Journals}

Édition électronique

URL : http://journals.openedition.org/questes/1054

DOI : 10.4000/questes. 1054

ISSN : 2109-9472

Éditeur

Les Amis de Questes

Édition imprimée

Date de publication : 15 septembre 2010

Pagination : $72-88$

ISSN : 2102-7188

\section{Référence électronique}

David Dominé-Cohn, « La gloire de Dieu, la gloire du prince. Manifestations divines et légitimation dans le duché de Bretagne au xive siècle », Questes [En ligne], 19 | 2010, mis en ligne le 01 janvier 2014, consulté le 22 septembre 2020. URL : http://journals.openedition.org/questes/1054 ; DOI : https:// doi.org/10.4000/questes.1054 


\title{
La gloire de Dieu, la gloire du prince. Manifestations divines et légitimation dans le duché de Bretagne au XIV ${ }^{\mathrm{e}}$ siècle
}

\author{
David DOMINE-COHN
}

«For what is he they follow? Truly, gentlemen, A bloody tyrant and a homicide ; (...)

Of England's chair, where he is falsely set;

One that hath ever been God's enemy. "

William SHAKESPEARE, Richard III, Acte V, scène 3 .

La bataille est le lieu d'un jugement, comme Shakespeare le montre vers 1591 à propos de la bataille de Bosworth Field en 1485 ; dans la nuit d'attente avant la bataille, les discours s'opposent avant les armes : d'un côté Richmond et de l'autre Richard. On trouve de fait une analogie évidente entre la bataille dans sa forme reconnue au Moyen Âge, celle de la confrontation de deux camps dans un choc frontal, et le duel autant judiciaire qu'ordalique. La mise en récit personnalise les camps, radicalise les questions posées, les enjeux et finalement construit une alternative sans voie médiane. Dans le cadre de ce schéma l'invocation de l'intervention divine devient possible : le vainqueur devient alors le choix de Dieu, et son existence même peut être considérée comme une manifestation divine.

La bataille est donc un moment théophanique, et la reprise par Shakespeare d'une telle construction nous invite alors à voir la force de ce schéma. Celui-ci fournit une clé de lecture efficace pour analyser les récits de bataille. Muni de cette clé, on s'intéressera à un épisode du XIV ${ }^{\mathrm{e}}$ siècle riche en batailles, la guerre de Succession de Bretagne entre 1341 et 1381. Cet épisode de l'histoire de Bretagne a fait l'objet de plusieurs mises en récit, en particulier dans la seconde moitié du $X V^{\mathrm{e}}$ siècle quand on voit apparaître à la cour du duc François II une série de chroniqueurs et 
d'historiens. Nous étudierons ici la première histoire bretonne de la guerre de Succession, celle qu'écrivit dans le Livre du Bon Jehan, duc de Bretagne, vers 1385, Guillaume de Saint-André ${ }^{1}$. Il s'agit d'une histoire quasi immédiate de la guerre de Succession, qui se termine en 1381 par le second Traité de Guérande en faisant définitivement droit à la famille de Montfort contre celle de Penthièvre. Sous la plume de Guillaume de SaintAndré, la bataille de l'Auray en 1364, qui oppose Jean de Montfort et Charles de Blois, tous les deux se revendiquant duc de Bretagne et successeur de Jean III mort en 1341, devient un jugement de Dieu entre deux chevaliers.

Nous nous proposons dans cette étude d'analyser les logiques à l'œuvre dans la mise en récit d'une manifestation divine dans le règlement d'un conflit de succession, qui se trouve aussi être un conflit pour le contrôle de l'entité politique assez considérable constituée par le duché de Bretagne. Il s'agit d'examiner au plus près d'une source émanant des milieux de la chancellerie ducale les modalités de la légitimation du pouvoir au milieu du XIV ${ }^{\mathrm{e}}$ siècle, en même temps que les usages politiques possibles des manifestations divines - ou du moins de leur récit.

Deux axes retiendront notre attention: d'une part une étude des modalités de la narration de l'intervention divine à la bataille de l'Auray en 1364 par Guillaume de Saint-André dans son Livre du Bon Jehan, duc de Bretagne, et, d'autre part, une étude du contexte religieux et politique qui anime le duché de Bretagne vers les années 1385, c'est-à-dire au moment de la rédaction du texte.

\footnotetext{
${ }^{1}$ Guillaume De Saint-Andre, Le Livre du Bon Jehan duc de Bretaigne, in JeanMichel Cauneau, Dominique PhilipPe (éd.), Chronique de l'État breton, Rennes, Presses Universitaires de Rennes, 2004, p. 224-549. Dans la suite de ce texte, nous y ferons référence par la mention Livre suivie des références aux vers cités.
} 


\section{Manifestations divines et légitimité ducale}

\section{Le récit : la place du merveilleux}

Guillaume de Saint-André accorde dans son Livre du Bon Jehan une part importante à la campagne victorieuse de 1362-1364 que conduit avec l'aide des Anglais Jean de Montfort, le futur duc Jean IV de Bretagne, contre le duc en titre et régnant Charles de Blois ${ }^{2}$. Dans ce cadre, le récit de la bataille de l'Auray en 1364 fait l'objet d'une construction narrative particulière. C'est au cours de cette bataille que Charles trouve la mort ; or, celle-ci est la condition presque suffisante à la reconnaissance de Jean comme duc de Bretagne. L'année 1363-1364 est construite par Guillaume autour de l'opposition des ruptures de trêves et des décisions d'offensive de Charles de Blois d'une part ${ }^{3}$, et de la préparation de Jean d'autre part ${ }^{4}$, suivie par sa victoire à la Lande d'Evran ${ }^{5}$. Pour cette bataille comme ce sera le cas pour celle de l'Auray, la description est sommaire, faisant de la bataille un événement pur, sans durée ni temporalité, manifestation immédiate du choix divin.

Guillaume fait de la bataille de l'Auray à l'automne 1364 l'occasion de présenter le rapport des princes à Dieu ${ }^{6}$. La préparation de la bataille est pour Jean, et selon Guillaume, une retraite en lui-même, entouré de ses amis $^{7}$. À l'inverse, Charles de Blois cherche à négocier encore. Son ubris le pousse à refuser les termes du traité préparé en 1363 et qui partageait la

\footnotetext{
${ }^{2}$ Le Livre compte 4164 vers (en comptant un prologue d'une centaine de vers). Sur ce total, la campagne de 1362-1364 compte près de 900 vers (des vers 519 aux vers 1446).

${ }^{3}$ Livre, vers 671-724 Charles rompt la trêve de Châteauneuf-de-la-Noue, conclue en 1362 et prend Trogoff et Béchere.

${ }^{4}$ Livre, vers 725-876 : Jean prépare ses troupes par un discours.

${ }^{5}$ Livre, vers 877-890.

${ }^{6}$ Ce sera de tout le Livre du Bon Jehan, duc de Bretaigne la seule mention d'une forme quelconque de dévotion.

${ }^{7}$ Essentiellement des chevaliers anglais qui commandent les troupes qui servent à la reconquête de la Bretagne part Jean; ce sont des alliés incontournables. "Et son estendart fist lever/ Et ses tentes pour soy loger/ la fut avecque luy Cliczon/ Chandoz, Latimer,et felton/ Et Quenolles et Kervalay », Livre, vers 999-1003.
} 
Bretagne entre les deux prétendants ${ }^{8}$. La décision d'aller à la bataille de l'Auray est donc clairement présentée comme l'œuvre de Charles de Blois. C'est lui encore qui force donc à la bataille un dimanche, contrevenant ainsi à toutes les règles de la chevalerie chrétienne ${ }^{9}$. Alors que la bataille ne peut plus être évitée, il s'active en préparatifs militaires quand Jean de Montfort, dans le récit de Guillaume de Saint-André, passe le temps qu'il lui reste en prière et en dévotion ${ }^{10}$. Dans ces conditions, la bataille de l'Auray est clairement présentée comme devenant l'affrontement de deux princes, l'un bon et l'autre mauvais, l'un dévoué à Dieu, et l'autre ne travaillant que pour son intérêt matériel immédiat et sa propre gloire. La réalité de ces comportements est tout à fait invérifiable : on sait seulement que Charles était un personnage très pieux, communiant tous les jours, se confessant tous les jours et souhaitant ne jamais commettre aucun péché, ainsi que la plupart des témoins l'ont affirmé pendant l'enquête en vue de sa canonisation, qui eut lieu entre 1369 et le milieu des années $1370^{11}$. Quant au comportement religieux de Jean de Montfort, aucun document n'en souligne de trait particulier. Il s'agit donc d'une stricte construction littéraire $^{12}$ qui a pour but de faire de la bataille, et de son résultat, une évidence et une preuve de l'action de Dieu dans le choix du vainqueur.

Le récit de la bataille est particulièrement court, surtout si on le

\footnotetext{
${ }^{8}$ Les vers 1180 à 1200 sont la réponse de Jean sur la question. On note que derrière cette attitude on retrouve celle de la bonne femme dans le jugement du Roi Salomon sur l'attribution d'un enfant à deux prostituées (Premier livre des Rois,3-16 à 3-28). Jean est donc considéré comme une bonne mère pour la Bretagne qui est une nouvelle fois présentée comme un tout indivisible.

${ }^{9}$ On retrouve ici le schéma classique dès l'époque de Guillaume de Saint-André de la bataille de Bouvines et qui a été analysé par Georges DuBY, in Le Dimanche de Bouvines, Paris, Gallimard, 1973.

${ }^{10}$ Vers 1244-1254.

11 Sur le modèle de piété de Charles de Blois, cf. André VAuchez La Sainteté en Occident aux derniers siècles du Moyen Âge, Rome, BEFAR, 1988 ( $2^{\text {ème }}$ éd.), p. 423 424.

${ }^{12}$ On peut même souligner qu'il s'agit de la seule référence faite au travers de toute l'œuvre de Guillaume de Saint-André à une forme de pratique religieuse de Jean.
} 
rapporte à l'ensemble du dispositif narratif qui raconte l'ensemble des préparatifs conduisant à cette bataille ${ }^{13}$. On peut donc s'interroger sur l'économie de l'épisode chez Guillaume. Celle-ci semble s'approcher de celle de l'hagiographie d'après la définition qu'en donne Alain Boureau dans son travail sur le récit chrétien au Moyen Âge, L'Événement sans $\mathrm{fin}^{14}$. Dans le chapitre I, «Le grand légendier chrétien », il signale que l'hagiographie et la legenda, tout en étant un «faisceau convergent de modes narratifs complexes $»^{15}$, n'en sont pas moins caractérisés par une construction très particulière de l'événement et de son récit. Il y a selon lui une tendance " au balisage thaumaturgique qui, au contraire, isole les nodularités sacrées ${ }^{16}$ pour mettre en scène l'apparition du divin dans les actions des hommes. Cette construction en alternance du récit entre narration chronologique, manifestation du choix divin et exégèse caractérise celle du Livre du Bon Jehan. Le récit de la campagne de 13631364 est construit autour de l'alternance de scènes de discours de Jean ou de Charles, de batailles, toutes présentées comme gagnées par Jean, et du récit de la progression de Jean de Montfort en Bretagne; le récit devient le moyen non pas de la construction de la légitimité de Jean mais la mise en évidence de celle-ci.

\footnotetext{
${ }^{13}$ Dans une certaine mesure on peut accepter l'idée que dans la narration de Guillaume de Saint-André, la bataille de l'Auray est l'aboutissement de toutes les entreprises conduites par Jean depuis son retour en Bretagne en 1363. Ce retour lui-même est présenté comme la première action faite et voulue par Jean, une fois devenu jeune homme. Toute la narration de Guillaume après le prologue (les vers 105 et suivants) est donc comme orientée vers cette bataille et son résultat.

${ }^{14}$ Alain Boureau, L'Événement sans fin. Récit et christianisme au Moyen Âge, Paris, Les Belles Lettres, 1993.

${ }^{15}$ Alain BoureAU, « Le grand légendier chrétien », in L'Événement sans fin, op. cit., p. 19.

${ }^{16}$ Ibid., p. 24.
} 


\section{La réception du récit}

Du côté de Jean de Montfort, on cherche immédiatement à faire de la bataille une œuvre de Dieu. Une lettre de Jean $\mathrm{IV}^{17}$ à son cousin le comte de Flandres, Louis II de Mâles, adopte les modalités narratives qu'on retrouvera chez Guillaume ${ }^{18}$. La lettre insiste sur le fait que la bataille a été voulue par Charles, et que celui-ci a donc été immédiatement sanctionné par Dieu, qui ne lui a pas donné la victoire et a même mis en déroute le restant des adversaires de Jean ${ }^{19}$. La lettre comprend aussi une demande de médiation entre Jean et le roi de France. Jean de Montfort souhaite faire l'hommage au roi pour le duché le plus vite possible ${ }^{20}$. L'intervention divine vient simplifier la pensée d'une succession problématique pour les Montfort. En effet, la crise de succession de Bretagne est ouverte à la mort

17 On connaît au moins trois lettres de Jean de Montfort à Louis de Mâles entre le 21 avril 1364 et le 8 octobre de la même année. À chaque fois, il s'agit de faire du puissant cousin par alliance le témoin des victoires et l'allié pour faire connaître les droits de Jean.

18 Il convient de se demander à la lecture de ces lettres si elles n'ont pas servi de base à Guillaume de Saint-André. En effet, il est plus que probable que ce dernier ait eu accès aux copies bretonnes de ces lettres pendant qu'il servait à la chancellerie ducale. Si Michael Jones, qui s'est chargé de l'édition des lettres de Jean IV (Michael JonES, Les Actes de Jean IV duc de Bretagne, Rennes, Presses Universitaires de Rennes, Klincksieck, 1981-1984, 3 volumes), ne signale qu'une référence dans les archives départementales du Nord: Lille, AD Nord B 18882 lettre missive fol 254, il ne mentionne aucune copie dans les archives départementales de Rennes ou de Nantes où on trouve les principaux actes ducaux. On peut cependant douter qu'aucune copie ne fût prise, malgré les conditions difficiles puisque la lettre est rédigée « sur les champs ».

19 «A vous savoir que le jour de la saint Michel derrein passé en plain champ aprés nos offres faiz de vouloir tracter de paiz et de bon accord o nostre feu adversaire môns. Charles de Bloys, et son refus sur ce lors et autrefoiz, Dieux par sa grâce nous donna la victoire sus nostre dit adversaire et sur sa compagnie qui estoint venuz nous assaillir sur nostre siege davent nostre chasteau Daurray qui nous fu auxi randu en retornement à nostre siege celui jour après la bataille. ", Lille, AD Nord B 18882 lettre missive fol 254, in Michael Jones, Les Actes de Jean IV, op. cit., $\mathrm{n}^{\circ} 37$.

20 «Et pour ce que Dieu a moustré nostre bon droit à qui le veut considérer nous envoions nos amez bacheliers môns. Jahen de Saint Gile et môns. Robert de Richer par devers nostre sire le Roy pour li expouser nostre voulonte et affection envers lui et les siens et de noz autres faiz et pour pourchacer nous avoir en sa recommandation et bonne grâce perseverable et nous recevoir en homme de nostre duché de bretaigne et nostre comté de Montfort et noz autre terre de France et requerre les nous délivrer selont la ténor de la paix et nous mectre en sauf respit si nostre presance y seroit nécessaire tant nous puissons aller seurement de vers lui et cessanz les perilz de chemins », idem. 
en 1341 du duc Jean III de Bretagne. Elle oppose d'une part Charles de Blois, mari de Jeanne de Penthièvre, fille de Jean III, et d'autre part Jean de Montfort, le père du futur duc Jean IV, vainqueur de la bataille qui nous préoccupe, lui-même neveu de Jean III. La revendication des deux protagonistes est fondée sur une référence à un mode de succession ${ }^{21}$. Charles s'appuie sur la coutume de Bretagne et soutient qu'il est possible que les femmes héritent du duché ; à l'inverse, en partant du principe que les pairies de France suivent la règle de France - i.e. : la loi salique nouvellement exhumée ${ }^{22}-$, Jean de Montfort soutient la stricte masculinité de la succession de Bretagne. Face à cette indécision, le roi de France saisit à la fin du printemps 1341 la cour des pairs pour trancher la question de droit. Une série d'exactions dans le duché durant l'été, principalement dues à Jean de Montfort, conduit le roi de France à transformer la procédure juridique; elle ne porte plus sur la validité d'un mode de succession mais tranche désormais la question de savoir qui le roi va recevoir comme son vassal pour le duché. Cette modification est une habile manœuvre de droit féodal qui permet au roi de mieux contrôler la succession de Bretagne, qui n'est plus considérée que sous l'angle de l'investiture féodale. Dans le même temps, ce déplacement d'enjeu va profondément modifier les fondements de l'autorité ducale dans le duché de Bretagne dans la mesure où il y a décorrélation de la question de la succession au duché et de l'accession à la couronne ducale, laissée finalement au seul choix du roi. C'est sur cette base que le roi de France entérine par l'arrêt de Conflans à

21 Arguments de Jean de Bretagne, comte de Montfort: Nantes, A.L.A., E 6 et A.N. J $241 n^{\circ} 44$. Argument de Charles de Blois: Paris, Bnf, MS fr. 18697 et 22338 (copies du XVI et XVII siècles).

22 On note deux éléments particulièrement intéressants. D'une part, la loi salique est une construction récente au moment du débat juridique de 1341. Cet usage nous renseigne donc sur le fait que le parti Montfort fait feu de tout bois. Dans le même temps, on constate une inversion remarquable puisque le parti soutenu par le roi de France en vient à soutenir une stricte succession par les femmes et le parti opposé au roi de France et doté de très forts liens avec le roi d'Angleterre, dont il soutient la cause dans la guerre de Cent ans, se trouve soutenir la « coutume de France» qui exclut les femmes. 
l'automne 1341 le choix de Charles de Blois en disqualifiant Jean de Montfort - père du futur Jean IV - comme possible vassal ${ }^{23}$.

Dans ce cadre, on comprend que la construction de la bataille de l'Auray en un jugement de Dieu entre deux candidats s'inscrit dans le cadre des pratiques de légitimation de Jean IV de Montfort. Le dispositif littéraire poursuit deux principaux objectifs à partir de la mise en scène de l'intervention divine. D'une part, on voit que la construction du récit autour de l'intervention fournit les moyens de penser l'éloignement du pouvoir ducal de la famille de Montfort. Jean de Montfort, père de Jean IV, s'est en effet trouvé très rapidement éloigné de la succession par l'action conjointe du roi de France, de Charles de Blois et d'une grande partie des seigneurs de Bretagne. Or, cet éloignement devient moins problématique si Dieu manifeste désormais explicitement son choix. D'autre part, l'intervention divine apparaît comme un argument juridique imparable dans la mesure où Dieu aurait exprimé sa préférence; le schéma de l'ordalie veut en effet que le vainqueur soit celui qui a le droit le plus fort. Par ailleurs Guillaume de Saint-André a radicalisé le résultat de la confrontation en plaçant l'ensemble des événements de son récit sous le signe d'une Fortune qui élève et qui abaisse, reprenant au passage le motif classique de la roue. Guillaume de Saint-André souligne ainsi le rôle de la Fortune, qui est ici un redresseur de tort dans la mesure où tous ceux qui possèdent des titres, et qui par leur comportement ne les méritent pas, se trouvent frustrés par elle de toutes leurs richesses et dignités ${ }^{24}$. La Fortune devient un instrument de Dieu contre ces derniers, qui se sont montrés pécheurs, dans la mesure où l'orgueil et l'absence de générosité sont des péchés.

23 L'argumentaire de l'arrêt, tout en soulignant les débats liés à la succession, porte avant tout sur la possibilité d'être reçu comme vassal.

${ }^{24}$ Livre, vers 45-46 et 51-55: « En chascun : « ren toy a moy :/ Je suy conte et duc et roy!/ (...) Affin que les riches soient clamés/ De toutes gens et redoubtez/ Mais certes Fortune leur fauldra/ De tout ce que leur promectra ». 
Le récit de la bataille de l'Auray et ses effets chez Guillaume de Saint-André forment donc un objet particulièrement cohérent et doté d'une grande force démonstrative. On comprend l'intérêt qu'il peut y avoir pour le duc de Bretagne, et pour les hommes de son entourage, de sa chancellerie, de sa cour, à pouvoir produire de tels discours. Cependant, on peut se demander quel intérêt particulier pousse à la rédaction d'une telle histoire un personnage comme Guillaume de Saint-André dans le courant des années 1380. En effet, à ce moment, le contexte politique du duché de Bretagne est profondément renouvelé après que les bases sur lesquelles Jean IV avait commencé son gouvernement en Bretagne ont été durement contestées. Ce rappel de l'élection quasi divine de Jean dans les années 1380, quand son pouvoir est déjà établi, ne doit pas cesser d'étonner.

\section{La place de Dieu dans l'espace politique du duché en 1385}

\section{Les nouvelles voies de légitimation du pouvoir}

Le Livre du Bon Jehan, duc de Bretagne est rédigé en 1385 par un membre important de la chancellerie ducale. Nous savons qu'il est un diplomate parfaitement au fait des principales affaires du duché ${ }^{25}$. L'importance que ce dernier accorde donc au récit de la vie de Jean de Montfort doit être soulignée, et l'attention qu'il porte à la bataille de l'Auray de 1364 est remarquable. Cette attention est remarquable dans la mesure où le moment supposé de la rédaction se situe dans les alentours de

\footnotetext{
${ }^{25}$ Les années 1990 et 2000 ont vu quelques études sur Guillaume de Saint-André. La première est celle de Michael JONES, «Un prince et son biographe : Jean IV, duc de Bretagne (1364-1399) et Guillaume de Saint-André », in Chantal GRELL, Werner PARAVICINI et Jürgen Voss (dir.), Les Princes et l'histoire du XIV au XVIII siècle. Actes du colloque organisé par l'Université de Versailles-Saint-Quentin et l'Institut historique allemand, Paris/Versailles, 13-16 mars 1996, Bonn, Bouvier (Pariser historische Studien, 47), 1998, p. 189-203. On citera ensuite une première étude de Jean-Michel CAuneau et Dominique PhiLIPPE, " Doubles jeux pour deux ducs et un chroniqueur. Louis $\mathrm{I}^{\mathrm{er}}$ d'Anjou, Jean IV de Bretagne, Guillaume de Saint-André et l'entrevue d'Angers (septembre 1381) », Archives d'Anjou, 6 (2002), p. 30-47.
} 
$1385^{26}$. À cette date, Jean IV de Montfort règne en Bretagne, avec la reconnaissance du roi de France à qui il a prêté hommage en 1381, depuis trois ans. Ce retour sur le trône est la conséquence d'une guerre conduite par Jean avec l'aide des Anglais contre les hommes du roi de France à partir de 1378 ; elle fait suite à l'exil du duc de Bretagne en 1373. À partir de cette date, on peut considérer que les modalités de légitimation et donc de production d'un discours sur la légitimité du duc ont profondément changé par rapport à la situation de 1364 qui était celle de la confrontation de deux candidats de droits prétendument égaux entre lesquels il fallait arbitrer pour trouver le bon duc.

Tant au vu des faits que du récit de Guillaume de Saint-André, de 1364 à 1373, le «bon duc » n'a pas su se faire aimer; si son exil est dû essentiellement à une révolte de Bretons excités par des envoyés du roi de France, Guillaume de Saint-André reconnaît aussi dans son récit que le gouvernement du duché ne va pas sans poser problème. Le constat d'un gouvernement ducal contesté est problématique dans une optique de légitimation du pouvoir en place. En effet, le mauvais gouvernement est le signe d'une déviance personnelle, souvent d'origine diabolique ${ }^{27}$. Dans ce cadre, une nouvelle manifestation divine ex nihilo serait sans doute problématique. Le signe sera donc la reconnaissance des mérites de Jean de Montfort, ces mérites relevant alors de la production d'une réforme personnelle. Guillaume réaffirme d'abord les qualités chevaleresques du duc; ce dernier en partant de Bordeaux et suivant une route dans le Périgord va entreprendre ce qui pourrait n'être qu'une chevauchée bretonne.

\footnotetext{
${ }^{26}$ Ce raisonnement vaut aussi si l'on suppose une date de rédaction postérieure à 1385 . On peut par ailleurs considérer qu'une rédaction très nettement antérieure à cette date n'est que très peu probable.

27 Sur cette question, on peut se rapporter aux nombreuses analyses faites sur les tyrannicides, et en particulier la thèse de Renaud VILLARD, Du bien commun au mal nécessaire - Tyrannies, assassinats politiques et souveraineté en Italie, vers 1470-vers 1600, Rome, École Française de Rome, 2008.
} 
Or, le récit de Guillaume de Saint-André va transformer cette entreprise en une recherche de la confrontation et de la bataille ${ }^{28}$. À Sarlat, la troupe épuisée, diminuée et sous-équipée de Jean rencontre les hommes de Jacob Laleulle. Face à l'épreuve, le courage renaît. Il s'agit du second grand récit de bataille du Livre du Bon Jehan duc de Bretaigne: il vient après l'Auray et est de fait le dernier. Le retour en Bretagne reste cependant particulièrement problématique : il ne peut en effet y avoir de retour par la force dans le duché, contre la volonté des sujets. C'est donc aux Bretons, vers l'année $1378^{29}$, que Guillaume attribue le premier geste, celui de se souvenir de Jean pour lutter contre les commissaires français ${ }^{30}$. Le fait que le duc de Bretagne soit nécessaire se révèle donc par le tort que cause son absence parmi les Bretons : son absence est définie comme un manque ${ }^{31}$. Il s'agit donc pour Guillaume de mettre en évidence un appel des seigneurs bretons à Jean, et cet appel participe d'un processus de remémoration de l'organisation féodale du duché et des fidélités dues au duc de Bretagne. En ce sens, il faut donc conclure que pour Guillaume de Saint-André les bases

${ }^{28}$ Plus généralement sur les formes de combats en cours à l'époque, voir Bernard GuEneE, La Guerre en Occident au Moyen Âge, Paris, Presses Universitaires de France, 1998.

${ }^{29}$ La chronologie de la révolte anti-française n'est pas précise chez Guillaume de SaintAndré. Ce manque de précision permet alors un effet littéraire qui accentue sa spontanéité.

30 « Aussi n'est cil qui ne s'avise/ Comment leur duc n'estoit pas mort/ Ainsi estoit hors a grant tort », vers 2996-2998.

${ }^{31}$ Il convient de se demander qui sont ces Bretons dont parle Guillaume de Saint-André. Nous reviendrons dans la seconde partie de ce chapitre sur le nationalisme supposé de Guillaume de Saint-André. Remarquons seulement que Guillaume définit la relation de ces Bretons au duc de Bretagne par le service qu'ils lui doivent : «Car ilz estoient touz obligez/ A le servir naturement/ Aussi avoint ilz fait serment/ D'estre o luy tous sans fantaisie » (vers 3004-3007. On note donc la récurrence du vocabulaire féodal : obligation, être à lui, serment. Ce vocabulaire peut nous conduire à croire - et cela a été le cas - que l'expression "les Bretons » désigne tous les Bretons et que cette situation décrite est in fine celle de tous les sujets du duc de Bretagne. Nous suivrons une hypothèse plus restreinte. Il s'agit bien plus probablement des seigneurs bretons, en particulier des fameux barons dont parle Guillaume de Saint-André. La vocation de ces vers est donc d'expliciter les relations complexes de Jean et de l'élite seigneuriale du duché. 
de l'exercice du pouvoir ducal dans le duché de Bretagne par Jean IV à partir de son retour en 1379 sont strictement féodales ${ }^{32}$. Ces liens et ces obligations existaient déjà, si l'on admet que Jean est déjà duc de Bretagne depuis au moins 1364 et la mort de Charles de Blois, si ce n'est 1344 et la mort de son père; mais l'urgence de la situation les rend concrètement nécessaires. La nécessité vient donc ici justifier la féodalité. Le registre du sentiment, de l'affection que doit un suzerain à ses vassaux est de mise pour décrire la réaction de Jean : «Le duc vit leur affection / Leur amour, leur dilection / et respondit courtoisement $\rangle^{33}$.

Dans tous les cas, les conditions politiques, diplomatiques et militaires du retour du duc, dont Guillaume se fait le narrateur précis, invalident en grande partie la thèse d'une élection divine comme suffisante pour faire valoir ses droits au duché, au profit d'une beaucoup plus efficace et immédiate élection par les pairs de Bretagne ${ }^{34}$. Jean de Montfort se voit donc bénéficier d'une double logique de légitimation au travers du récit des évènements de sa vie. La première logique de légitimation est celle qui vient de Dieu. Elle est manifeste dès 1364 et porte d'abord sur la désignation de Jean contre Charles de Blois. La seconde légitimation vient de l'élite du duché de Bretagne et est bien postérieure dans la mesure où elle ne date que de 1378. Ce décalage dans le temps est problématique dans la mesure où la désignation par Dieu pourrait sembler à première vue non

\footnotetext{
${ }^{32}$ Le récit de la rencontre sur la grève de Bretagne de Jean IV et des seigneurs bretons est directement présentée comme une cérémonie d'hommage féodale : « Si se vont touz agenoiller,/ Par devant lui sur le gravier/ Et disoint : Nostre droit seignour,/ Aujourd'hui vous doint Dieu bon jour/ Et vous garde de vilain reproche./ Adonc envers eulx il s'approche/ Et les va lever doulcement/ Et les besa en soupirant/ Et les salua touz ensemble/ Et si ploura comme il me semble/ De grand amance qu'au cueur avoit/ Quant devant lui la les avoit », vers 3053-3064.

${ }^{33}$ Vers 3025-3027.

34 Voir le récit proposé par Guillaume de Saint-André d'abord du constat fait par les Bretons face à l'occupation française, du retour, de l'accueil et de la nouvelle unité politique formée entre le duc reconnu par les Bretons et les seigneurs bretons (en particulier l'épisode de la fondation de l'ordre de l'hermine).
} 
suffisante, cependant cette double légitimité et sa mise en scène sont nécessaires pour représenter non seulement le pouvoir, mais aussi l'autorité dont Jean de Montfort est investi comme duc de Bretagne dans les années 1380 .

On observe un changement notable entre le récit des événements avant 1373 et après 1373. Dans le premier temps, Jean est l'instrument de Dieu, dans une phase du récit qui correspond à un manque de droit - l'effet rhétorique de l'alternance d'épisodes de la vie de Jean et de digressions moralisantes ou juridiques de Guillaume, stratégie de contournement de la question du droit qui permet de maintenir une évidence de la présence, peut être interprété dans ces termes. Inversement, après les années 1373, Jean a tendance à disparaître sensiblement du récit au profit d'autres personnages ou bien de plans beaucoup plus larges, qui font la place à d'autres acteurs comme lui connotés positivement ${ }^{35}$. La logique du récit serait donc de mettre en scène une dynamique dont Jean serait une fois encore l'instrument, et qui aurait pour origine la nécessité de maintenir le duché de Bretagne. La nécessité de maintenir le duché contient en soi une dynamique de solidarité et d'autorité autour du duché comme institution stable et faisant référence pour tous les acteurs; elle vient compléter la première source de légitimation qu'avait été la manifestation du choix de Dieu à la bataille de l'Auray. Le bon gouvernement étant à son tour nécessairement une manifestation divine, on assiste donc à un réinvestissement religieux du politique dans le duché de Bretagne. Il convient maintenant d'analyser quelques pistes pour comprendre les logiques et les usages de ce réinvestissement.

\footnotetext{
${ }^{35}$ On pense en particulier à la joyeuse entrée de Jean IV dans la ville de Dinan en 1379 , relatée aux vers 3065-3094.
} 


\section{Le réinvestissement religieux du politique dans le duché}

On connaît la place prépondérante des structures religieuses dans l'organisation de la société occidentale au Moyen Âge; il n'y a pas de déconnexion entre l'espace religieux et l'espace politique et social ${ }^{36}$ et tout particulièrement pour le duché de Bretagne. Les réseaux de monastères et de couvents y sont particulièrement dynamiques, mais surtout l'emprise des évêques est très forte. On retient avec Jean-Pierre Leguay ${ }^{37}$ l'importance du réseau des villes épiscopales dans le duché et donc le poids politique et social des prélats bretons. À l'occasion de la guerre de Succession, le soutien des évêques à Charles de Blois fut particulièrement déterminant et ne permit pas au père de Jean IV de tenir suffisamment fort le duché pour asseoir une domination telle qu'elle aurait pu servir de base à une négociation avec le pouvoir royal. C'est d'ailleurs parmi les évêques qu'on trouvera jusque dans les années 1380 les plus vives oppositions à la présence des Montfort sur le trône de Bretagne. Ainsi, jusqu'en $1384^{38}$, l'évêque de Saint-Malo, Josselin de Rohan, par ailleurs membre de l'une des principales familles aristocratiques du duché, conduit une lutte féroce contre Jean IV qu'il n'a jamais reconnu comme duc de Bretagne et plus particulièrement comme co-seigneur de la cité avec lui. Cette opposition du

\footnotetext{
${ }^{36}$ Nous nous rapportons aux études récentes proposées dans Florian MAZELL (dir.), $L$ 'Espace du diocèse. Genèse d'un territoire dans l'Occident médiéval ( $V^{e}$-XIII siècle), Rennes, Presses Universitaires de Rennes, 2008.

37 Jean-Pierre Leguay, Un Réseau urbain au Moyen Âge. Les villes du duché de Bretagne aux XIV et $X V^{e}$ siècles, Paris, Maloine, 1981, et Vivre dans les villes bretonne au Moyen Âge, Rennes, Presses Universitaires de Rennes, 2009.

${ }^{38}$ Nous connaissons la lettre de rémission que Jean accorde à l'évêque et aux bourgeois de la ville pour leur conduite (Nantes, A.LA., E $159 n^{\circ}$ ). Dans le cadre de celle-ci le duc emploie pour la première fois le terme de lèse-majesté. $\mathrm{Si}$ on reprend la construction canonique du crime de lèse-majesté telle qu'elle fut exposée en particulier par Jacques Chiffoleau, la lèse-majesté est avant tout la conséquence d'un crime religieux comme 1'hérésie. Sans postuler à partir de là que le duc de Bretagne accuse formellement Josselin de Rohan d'hérésie, nous pouvons interpréter l'usage de ce vocable particulièrement fort comme une tentative ducale de reprise en main des processus juridiques et politiques à l'œuvre dans le cadre de ce conflit entre le duc de Bretagne et un évêque de Bretagne.
} 
prélat a commencé avec la demande par Jean d'un fouage exceptionnel en 1366 ; elle se poursuit par tous les moyens à la disposition du prélat dont l'appel au Saint-Siège. L'arrivée du Pape dans le conflit n'est pas anodine : les années 1360 et surtout 1370 sont l'occasion d'une série de confrontations entre le duc de Bretagne et les différents évêques de Rome, en particulier Grégoire XI. Au-delà du cas de l'évêque de Saint-Malo qui reste marginal, la principale source de litige entre l'Église et Jean de Montfort dans le duché de Bretagne reste le procès en canonisation dont Charles de Blois fait l'objet à partir de 1369.

Les relations entre le duc de Bretagne et le Saint-Siège sont particulièrement complexes. Un contentieux oppose en particulier le duc Jean IV de Montfort et le pape Grégoire XI. Celui-ci soutient la canonisation de Charles de Blois, qui au début des années 1370 est considérée comme acquise, l'extinction de la procédure n'ayant lieu que dans les années 1380. En effet, après sa mort à la bataille de l'Auray en 1364, Charles de Blois fut inhumé dans le couvent des Franciscains de Guingamp. Dans le courant des années 1360 on rapporte que sa tombe est l'objet de dévotions de bandes d'enfants venus d'abord du Poitou, puis de la Bretagne ${ }^{39}$. À cette occasion, le souvenir collectif de Charles se transforme. Charles devient un dévot et un saint homme, alors qu'il était considéré en particulier par les aristocrates bretons comme bigot, et faible devant les demandes de l'Église ${ }^{40}$.

Le culte rendu à Charles de Blois fait d'abord l'objet d'une condamnation par la bulle Molesta significatio (15 X 1368), mais dont l'envoi aux évêques et au clergé de Bretagne est incertain. Urbain V

\footnotetext{
39 Jean Delalande, Les extraordinaires croisades d'enfants et de pastoureaux au Moyen Âge. Les pèlerinages d'enfants au Mont Saint-Michel, Paris, Lethielleux, 1961, p. 168.

40 André Vauchez, La Sainteté en Occident aux derniers siècles du Moyen Âge, op. cit., p. 424.
} 
intervient pour condamner le culte rendu à Charles de Blois. Le pape condamne en particulier le culte rendu à une image de Charles dans l'église des cordeliers de Dinan qui, lorsque Jean IV aurait demandé son effacement, se serait mise à saigner. Mais l'année suivante un procès est officiellement ouvert à Angers à la demande de Louis d'Anjou, neveu de Charles et membre de la famille royale française. Une partie des évêques bretons et surtout des Frères Mineurs soutiennent activement la procédure. Ce procès est l'occasion de la refondation des réseaux de soutien à la cause des Blois-Penthièvre sous l'égide de la duchesse douairière Jeanne, veuve de Charles, et de leur jeune fils. Dans le même temps, le procès en canonisation alimente l'idée d'un martyre de Charles $^{41}$. Cette idée s'affirme dans les années 1370 quand justement la contestation de la politique de Jean IV se fait de plus en plus ouverte, à l'occasion par exemple de la sécession de Saint-Malo qui s'offre à Grégoire XI. Mort et en voie de canonisation, Charles de Blois reste un rival gênant d'autant que sa nouvelle réputation annule en grande partie les effets légitimateurs de la bataille de l'Auray.

Dans ce contexte, la rédaction par Guillaume de Saint-André d'un récit ordalique à la bataille de l'Auray en 1364, réaffirmant la manifestation divine originelle qui a permis l'arrivée au pouvoir de Jean, ne doit pas tant être comprise comme un discours sur les fondements objectifs du pouvoir ducal, ni même sur les modalités particulières de l'autorité ducale dans le duché de Bretagne mais bien comme la reprise en main des discours sur les phénomènes religieux survenus dans le duché de Bretagne depuis la mort de Jean III en 1341. À la narration de la vie de Charles de Blois qui est proposée dans le cadre de la rédaction des actes de son procès on en

${ }^{41}$ Antoine de SÉRENT, « Charles de Blois, duc de Bretagne (1319-1364) et l'Ordre de Saint François », Études Franciscaines, 8 (1957), p. 59-75 et Hervé MarTin, Les Ordres mendiants en Bretagne (env. 1230-1530). Pauvreté volontaire et prédication à la fin du Moyen Âge, Paris, Klincksieck, 1975, p. 366-371 et p. 409-411. 
opposer une autre, contrôlée, favorable au duc de Bretagne en place. On assiste alors à une démarche de constitution d'un monopole de la manifestation divine et légitime dans le duché de Bretagne.

À rebours d'une lecture des évolutions politiques des $\mathrm{XIV}^{\mathrm{e}}$ et $\mathrm{XV}^{\mathrm{e}}$ siècles qui en font les siècles de la rationalisation progressive des instruments de pouvoir avant la constitution d'un espace de gouvernement objectivé $^{42}$, il convient donc de faire de l'imaginaire politique médiéval un objet complexe fait de nombreuses dimensions dont non seulement celles que nous qualifierions de religieuses mais aussi comprenant des aspects merveilleux.

Avant les études de Jacques Le Goff, qui ont souligné toute l'importance du merveilleux dans l'imaginaire médiéval - même le plus savant -, Marc Bloch dans sa thèse sur les Rois thaumaturges avait souligné les relations fondamentales entre magie et pouvoir au Moyen $\hat{A} g e^{43}$. Les pouvoirs merveilleux du prince sont les signes de son élection par Dieu, de sa légitimité affirmée par Dieu, en même temps que la démonstration de la gloire de Dieu.

42 Jean KerherVE, L'État breton aux XIVe et XVe siècles, les ducs, l'argent, les hommes, Paris, Maloine, 1987, 2 volumes.

${ }^{43}$ Marc Bloch, Les Rois thaumaturges, Paris, Gallimard, 1983 ( $1^{\text {ère }}$ édition en 1924), p. 185 sqq. 